\title{
Macroscale boundary conditions for a non-linear heat exchanger
}

\author{
Chen Chen ${ }^{1} \quad$ A. J. Roberts ${ }^{2} \quad$ J. E. Bunder ${ }^{3}$
}

(Received 8 April 2015; revised 19 September 2015)

\begin{abstract}
Multiscale modelling methodologies build macroscale models of materials with complicated fine microscale structure. We propose a methodology to derive boundary conditions for the macroscale model of a prototypical non-linear heat exchanger. The derived macroscale boundary conditions improve the accuracy of the macroscale model. We verify the new boundary conditions by numerical methods. The techniques developed here can be adapted to a wide range of multiscale reaction-diffusion-advection systems.
\end{abstract}

http://journal.austms.org.au/ojs/index.php/ANZIAMJ/article/view/9528 gives this article, (C) Austral. Mathematical Soc. 2015. Published September 29, 2015, as part of the Proceedings of the 17th Biennial Computational Techniques and Applications Conference. ISSN 1446-8735. (Print two pages per sheet of paper.) Copies of this article must not be made otherwise available on the internet; instead link directly to this URL for this article. 


\section{Contents}

1 Introduction

2 A normal form of the spatial evolution

3 Projection reveals boundary conditions

4 A numerical example

C26

5 Conclusion

C28

\section{Introduction}

Multiscale modelling techniques are a developing area of research in engineering and physical sciences. These techniques are needed when the system being modelled possesses very different space-time scales and it is infeasible to simulate the whole domain on a microscale mesh (Dolbow et al. 2004, Kevrekidis and Samaey 2009, Bunder \& Roberts 2012). But macroscale boundary conditions are rarely derived systematically; instead macroscale boundary conditions are often proposed heuristically (Pavliotis and Stuart 2008, Mei and Vernescu 2010, Mseis 2010). We developed a systematic method to derive boundary conditions for one-dimensional linear problems with fine structure by cell mapping (Chen et al. 2014). Here we extend the method to a prototypical non-linear heat exchanger problem.

We mathematically model the counter flow two-stream heat transfer shown in Figure 1. Let $x$ measure nondimensional distance along the heat exchanger which is of length $\mathrm{L}, 0 \leqslant x \leqslant \mathrm{~L}$, and let $\mathrm{t}$ denote nondimensional time. The field $a(x, t)$ is the temperature of the fluid in one pipe and field $b(x, t)$ is that in the other pipe. A quadratic reaction is included as an example nonlinearity 
Figure 1: A schematic diagram of a heat exchanger. The red pipeline carries fluid to the right, and the blue pipeline carries fluid to the left. Heat exchanges between the pipes.

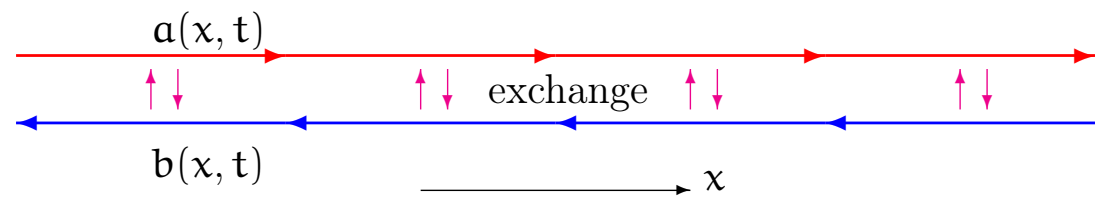

to give the nondimensional microscale PDEs

$$
\begin{aligned}
& \frac{\partial a}{\partial t}=\frac{1}{2}(b-a)+\frac{1}{2} a^{2}-\frac{\partial a}{\partial x}+3 \frac{\partial^{2} a}{\partial x^{2}}, \\
& \frac{\partial b}{\partial t}=\frac{1}{2}(a-b)-\frac{1}{2} b^{2}+\frac{\partial b}{\partial x}+3 \frac{\partial^{2} b}{\partial x^{2}} .
\end{aligned}
$$

The lateral diffusion also included in these PDEs makes the derivation of macroscale boundary conditions challenging.

Various mathematical methodologies derive from PDEs (1) the macroscale model

$$
\frac{\partial C}{\partial t}=\frac{1}{2} C^{3}-2 C \frac{\partial C}{\partial x}+4 \frac{\partial^{2} C}{\partial x^{2}}+\mathcal{O}\left(C^{4}+\partial_{x}^{4}\right),
$$

for the mean temperature $C(x, t):=[a(x, t)+b(x, t)] / 2$. For example, centre manifold theory rigorously derives this effective macroscale model (Roberts 2013), as does homogenization (Pavliotis and Stuart 2008, Mei and Vernescu 2010). This macroscale model combines an effective cubic reaction, with an effective nonlinear advection, and enhanced lateral diffusion. The necessary analysis to derive the model (2) is based around the equilibrium $a=b=0$, and applies to the slowly-varying in space solutions in the interior of the domain. For example, in the interior it predicts the temperature fields are

$$
\left[\begin{array}{l}
a \\
b
\end{array}\right]=C\left[\begin{array}{l}
1 \\
1
\end{array}\right]+\left(\frac{1}{2} C^{2}-\frac{\partial C}{\partial x}\right)\left[\begin{array}{c}
1 \\
-1
\end{array}\right]+\mathcal{O}\left(C^{3}+\partial_{x}^{3}\right) .
$$

The challenge of this article is to provide sound boundary conditions for the macroscale model (2). 
Prototypical microscale boundary conditions for microscale system (1) are taken to be the Dirichlet boundary conditions

$$
a(0, t)=a_{0}, \quad b(0, t)=b_{0}, \quad a(L, t)=a_{L}, \quad \text { and } \quad b(L, t)=b_{L},
$$

where $a_{0}, a_{L}, b_{0}$ and $b_{L}$ are potentially slowly varying functions of time. Section 3 derives nonlinear macroscale boundary conditions (12) for the macroscale mean temperature model (2). For example, the linearisation of the macroscale boundary condition (14) derived from the Dirichlet boundary conditions (4) is the Robin condition

$$
C-\frac{1}{2} \frac{\partial C}{\partial x} \approx \frac{1}{4} b_{0}+\frac{3}{4} a_{0} \text { at } x=0 .
$$

Importantly, this is not a Dirichlet boundary condition despite the microscale boundary conditions and the definition $C:=(a+b) / 2$ together suggesting boundary conditions are (incorrectly) $C(0, t)=\left(a_{0}+b_{0}\right) / 2$.

Figure 2 plots microscale and macroscale solutions for the heat exchanger at a particular time. The two solid lines plot the microscale solution $a(x, t)$ and $b(x, t)$ of microscale PDE (1) with microscale boundary conditions (4). The black dashed line plots the mean temperature model (2) with classic Dirichlet boundary conditions $C(0, t)=\left(a_{0}+b_{0}\right) / 2$ and $C(L, t)=$ $\left(a_{L}+b_{L}\right) / 2$ as would be commonly invoked (Mei and Vernescu 2010, Mseis 2010, Ray et al. 2012). The macroscale model (2) performs poorly with these heuristic Dirichlet boundary conditions, especially in the interior of the domain (here $5 \leqslant x \leqslant 25$ ). But the interior is where the macroscale model (2) should be valid. The macroscale model (2) represents the interior dynamics but cannot resolve the details of boundary layers (Roberts 1992). With our derived boundary conditions, the macroscale solution (red line in Figure 2) fits the microscale solution (solid lines) in the interior: the microscale fields $a(x, t)$ and $b(x, t)$ being given by equation (3). Our systematic derivation of boundary conditions is needed for macroscale models to correctly predict the interior dynamics.

The key to our approach is to explore the effect of boundary layers by treating space as a time-like variable (e.g., Chen et al. 2014). However, the heat 
Figure 2: Example solutions of the heat exchanger (1) in domain $0 \leqslant x \leqslant$ $\mathrm{L}=30$ at time $\mathrm{t}=21$. The two solid lines plot the the temperature of the two pipes, $a(x, t)$ and $b(x, t)$. The dashed lines are solution of the macroscale model (2) at $t=21$ : (black dashed) with heuristic Dirichlet boundary conditions; and (red dash-dots) with our systematically derived boundary conditions.

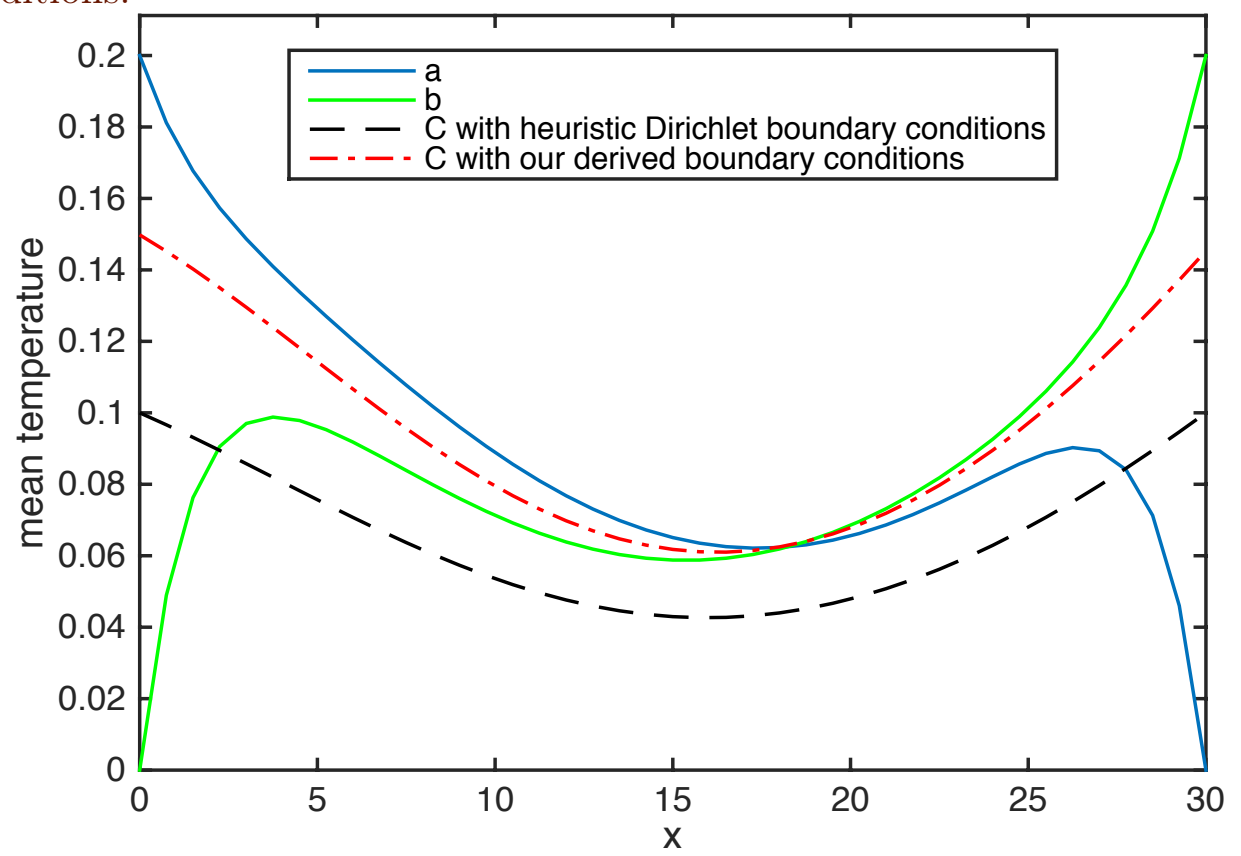


exchanger problem (1) is challenging because of the nonlinearity. Here, a normal form coordinate transformation separates the spatial evolution in the boundary layers into a slow manifold, stable manifold and unstable manifolds. This separation empowers a transformation of the given physical boundary conditions (4) into boundary conditions (13) for the macroscale interior model (2).

\section{A normal form of the spatial evolution}

The macroscale model (2) is slow so the dominant terms in the boundary layers are due to the derivatives of spatial structure. Thus, to derive macroscale boundary conditions for slow evolution (2) we treat the time derivative $\partial / \partial t$ as a negligible operator (Roberts 1992). To put heat exchanger system (1) into the form of a dynamical system in time-like variable $x$ we define $a^{\prime}:=\frac{\partial a}{\partial x}$ and $b^{\prime}:=\frac{\partial b}{\partial x}$. Then rearranging system (1) into dynamical system form, with $\partial_{t}=0$ for quasi-steady solutions, gives

$$
\frac{\partial}{\partial x}\left[\begin{array}{c}
a \\
b \\
a^{\prime} \\
b^{\prime}
\end{array}\right]=\left[\begin{array}{cccc}
0 & 0 & 1 & 0 \\
0 & 0 & 0 & 1 \\
\frac{1}{6} & -\frac{1}{6} & \frac{1}{3} & 0 \\
-\frac{1}{6} & \frac{1}{6} & 0 & -\frac{1}{3}
\end{array}\right]\left[\begin{array}{c}
a \\
b \\
a^{\prime} \\
b^{\prime}
\end{array}\right]+\left[\begin{array}{c}
0 \\
0 \\
-\frac{1}{2} a^{2} \\
\frac{1}{2} b^{2}
\end{array}\right] .
$$

We analyse the (spatial) dynamics of this system with 'initial condition' at $x=0$ of the given microscale Dirichlet boundary conditions (4).

Start by basing the analysis of (5) around the equilibrium at the origin, $a=b=a^{\prime}=b^{\prime}=0$. The eigenvalues of the system linearised about the origin are 0 (twice) and $\pm \sqrt{2}$. The eigenvalues of zero correspond to an eigenvector of $(1,1,0,0)$ and a generalised eigenvector of $(-1,1,1,1)$. Hence the spatial ODE system (5) contains two centre (slow) modes, one stable mode and one unstable mode.

Roberts $(2014 a$,$) provides a web service to construct by computer algebra a$ coordinate transform which separates stable, unstable and centre manifolds. 
However, the web service does not directly apply to systems whose linearisation has a generalised eigenvector. To circumvent the generalised eigenvector, we choose to embed the ODE system (5) as the $\epsilon=1$ member of the one parameter family of systems

$$
\frac{\partial}{\partial x}\left[\begin{array}{c}
a \\
b \\
a^{\prime} \\
b^{\prime}
\end{array}\right]=\left[\begin{array}{cccc}
0 & 0 & 1 & -1 \\
0 & 0 & -1 & 1 \\
\frac{1}{6} & -\frac{1}{6} & -\frac{1}{6} & \frac{1}{2} \\
-\frac{1}{6} & \frac{1}{6} & -\frac{1}{2} & \frac{1}{6}
\end{array}\right]\left[\begin{array}{c}
a \\
b \\
a^{\prime} \\
b^{\prime}
\end{array}\right]+\left[\begin{array}{c}
\epsilon b^{\prime} \\
\epsilon a^{\prime} \\
-\frac{1}{2} a^{2}+\frac{1}{2} \epsilon a^{\prime}-\frac{1}{2} \epsilon b^{\prime} \\
\frac{1}{2} b^{2}+\frac{1}{2} \epsilon a^{\prime}-\frac{1}{2} \epsilon b^{\prime}
\end{array}\right],
$$

where the last vector on the right-hand side is treated as a perturbative term: the parameter $\epsilon$ counts the order of artificial linear perturbation. The linear operator in system (6) now has no generalised eigenvector: its eigenvalues are 0 (twice) and $\pm \frac{2}{3}$, with corresponding eigenvectors $(1,1,0,0)$, $(-1,1,1,1),\left(-\frac{3}{2}, \frac{3}{2}, 0,1\right),\left(-\frac{3}{2}, \frac{3}{2}, 1,0\right)$. The web service (Roberts 2014a) then finds a normal form coordinate transform as a multivariate power series in variables $s_{j}$ and parameter $\epsilon$. Substituting $\epsilon=1$ into the results reveals the centre manifold, stable manifold and unstable manifold for the spatial ODE (5).

The three manifolds can be parametrised as we choose. We choose the definition of the two parameters for the slow manifold to be the mean temperature $s_{1}=C$ and its spatial derivative $s_{2}=\frac{\partial C}{\partial x}$ :

$$
\begin{array}{ll}
s_{1}:=\frac{1}{2}(a+b)=C, & s_{3}:=\frac{1}{8}\left(3 a-3 b-3 a^{\prime}+9 b^{\prime}\right), \\
s_{2}:=\frac{1}{2}\left(a^{\prime}+b^{\prime}\right)=\frac{\partial C}{\partial x}, & s_{4}:=\frac{1}{8}\left(3 a-3 b+9 a^{\prime}-3 b^{\prime}\right),
\end{array}
$$

where $s_{3}$ parametrises the stable manifold, and $s_{4}$ parametrise the unstable manifold. Then the web service (Roberts 2014a) derives the coordinate transform (8) giving $a, b, a^{\prime}$ and $b^{\prime}$ as power series of $s_{1}, s_{2}, s_{3}, s_{4}$ and $\epsilon$. 
When $\epsilon=1$ the power series are

$$
\begin{aligned}
\mathrm{a} \approx & \mathrm{s}_{1}-\mathrm{s}_{2}+0.25 \mathrm{~s}_{3}+1.5 \mathrm{~s}_{1}^{2}+6 \mathrm{~s}_{2}^{2}-1.1 \mathrm{~s}_{1} \mathrm{~s}_{3}-3.4 \mathrm{~s}_{2} \mathrm{~s}_{3}-0.035 \mathrm{~s}_{3}^{2} \\
& +0.75 \mathrm{~s}_{4}+0.74 \mathrm{~s}_{2} \mathrm{~s}_{4}+0.56 \mathrm{~s}_{3} \mathrm{~s}_{4}-0.25 \mathrm{~s}_{4}^{2} \\
\mathrm{~b} \approx & \mathrm{s}_{1}+\mathrm{s}_{2}-0.75 \mathrm{~s}_{3}-1.5 \mathrm{~s}_{1}^{2}-6 \mathrm{~s}_{2}^{2}-0.74 \mathrm{~s}_{2} \mathrm{~s}_{3}+0.25 \mathrm{~s}_{3}^{2} \\
& \quad-0.25 \mathrm{~s}_{4}-1.1 \mathrm{~s}_{1} \mathrm{~s}_{4}+3.4 \mathrm{~s}_{2} \mathrm{~s}_{4}-0.56 \mathrm{~s}_{3} \mathrm{~s}_{4}-0.035 \mathrm{~s}_{4}^{2} \\
\mathrm{a}^{\prime} \approx & \mathrm{s}_{2}+1.5 \mathrm{~s}_{1} \mathrm{~s}_{2}-0.17 \mathrm{~s}_{3}+0.56 \mathrm{~s}_{1} \mathrm{~s}_{3}+0.91 \mathrm{~s}_{2} \mathrm{~s}_{3}+0.47 \mathrm{~s}_{3}^{2} \\
& +0.5 \mathrm{~s}_{4}-0.56 \mathrm{~s}_{1} \mathrm{~s}_{4}+1.2 \mathrm{~s}_{2} \mathrm{~s}_{4}-0.33 \mathrm{~s}_{4}^{2} \\
\mathrm{~b}^{\prime} \approx & \mathrm{s}_{2}-1.5 \mathrm{~s}_{1} \mathrm{~s}_{2}+0.5 \mathrm{~s}_{3}+0.56 \mathrm{~s}_{1} \mathrm{~s}_{3}+1.2 \mathrm{~s}_{2} \mathrm{~s}_{3}-0.33 \mathrm{~s}_{3}^{2} \\
& -0.17 \mathrm{~s}_{4}-0.56 \mathrm{~s}_{1} \mathrm{~s}_{4}+0.91 \mathrm{~s}_{2} \mathrm{~s}_{4}-0.047 \mathrm{~s}_{4}^{2}
\end{aligned}
$$

Evaluating the power series at $\epsilon=1$ recovers the coordinate transform applicable to the original spatial system (5). For simplicity we only record these and later expressions correct to quadratic terms in $s_{j}$, that is, with cubic errors in the multinomial, and we record coefficients to two significant figures. The corresponding evolution of the spatial system (5) in these new variables $s_{j}$ is also provided by the web service which determines

$$
\begin{aligned}
& \frac{\partial s_{1}}{\partial x} \approx s_{2}, \\
& \frac{\partial s_{2}}{\partial x} \approx 1.5 s_{1} s_{2}, \\
& \frac{\partial s_{3}}{\partial x} \approx-0.67 s_{3}-0.75 s_{3} s_{1}-0.94 s_{3} s_{2}, \\
& \frac{\partial s_{4}}{\partial x} \approx+0.67 s_{4}-0.75 s_{4} s_{1}+0.94 s_{4} s_{2} .
\end{aligned}
$$

The normal form of the transformed system (9) has useful properties. Since $\frac{\partial s_{3}}{\partial x}=g_{3}\left(s_{1}, s_{2}\right) s_{3}$ and $\frac{\partial s_{4}}{\partial x}=g_{4}\left(s_{1}, s_{2}\right) s_{4}$ for some functions $g_{j}$, the three invariant manifolds of the system (9) are $s_{3}=0, s_{4}=0$ and $s_{3}=s_{4}=0$. From the linearisation of (9), these are the centre-unstable, centre-stable, and slow manifolds, respectively. Further, because $\frac{\partial s_{1}}{\partial x}$ and $\frac{\partial s_{2}}{\partial x}$ are functions of only $s_{1}$ and $s_{2}$, the planes of $s_{1}$ and $s_{2}$ constant are isochrons of the slow 
manifold (Roberts 1989) (sometimes called the leaves of the foliation, fibres, a fibration, fibre maps or fibre bundles (e.g., Murdock 2003, pp.300-2)).

One might query whether the transformation (8) and (9) is valid given that it is obtained by a power series in artificial parameter $\epsilon$ that is then evaluated at $\epsilon=1$. The coefficients appear to converge well to the given values, but as an independent check we also embedded the spatial ODE (5) into the different family of problems

$$
\frac{\partial}{\partial x}\left[\begin{array}{c}
a \\
b \\
a^{\prime} \\
b^{\prime}
\end{array}\right]=\left[\begin{array}{cccc}
0 & 0 & 1 & -1 \\
0 & 0 & -1 & 1 \\
\frac{1}{6} & -\frac{1}{6} & \frac{1}{6} & \frac{1}{6} \\
-\frac{1}{6} & \frac{1}{6} & -\frac{1}{6} & -\frac{1}{6}
\end{array}\right]\left[\begin{array}{c}
a \\
b \\
a^{\prime} \\
b^{\prime}
\end{array}\right]+\left[\begin{array}{c}
\epsilon b^{\prime} \\
\epsilon a^{\prime} \\
-\frac{1}{2} a^{2}+\frac{1}{6} \epsilon a^{\prime}-\frac{1}{6} \epsilon b^{\prime} \\
\frac{1}{2} b^{2}+\frac{1}{6} \epsilon a^{\prime}-\frac{1}{6} \epsilon b^{\prime}
\end{array}\right]
$$

Performing the same algebraic construction, but from this quite different base, we find system (10) results in the same transform (8) and evolution (9). This confirms the perturbative approach via embedding.

\section{Projection reveals boundary conditions}

This section focuses on the boundary layer near $x=0$. As shown by the solid lines in Figure 2, the microscale boundary conditions at $x=0$ force a boundary layer in the microscale model (1). However, the macroscale model (2) does not resolve the boundary layer. Forcing the macroscale model to pass through $\left(a_{0}+b_{0}\right) / 2$ introduces an error in the interior of the domain, as shown by the dashed black line in Figure 2. Here we derive an improved boundary condition at $x=0$ which reduces the interior error caused by the poorly chosen macroscale boundary condition.

The boundary layer must lie in the centre-stable manifold $s_{4}=0$ because if there was any component $s_{4} \neq 0$, then this would grow exponentially quickly in space and dominate the solution across the whole domain. Algebraically we obtain the centre-stable manifold by substituting $s_{4}=0$ into the coordinate 
Figure 3: Schematic plot of centre-stable manifold near the boundary $x=0$. The green plane is the centre manifold. The blue solid line is the set of values allowed by the microscale boundary condition at $x=0$. The blue dotted line is the projection of the microscale boundary values onto the slow manifold.

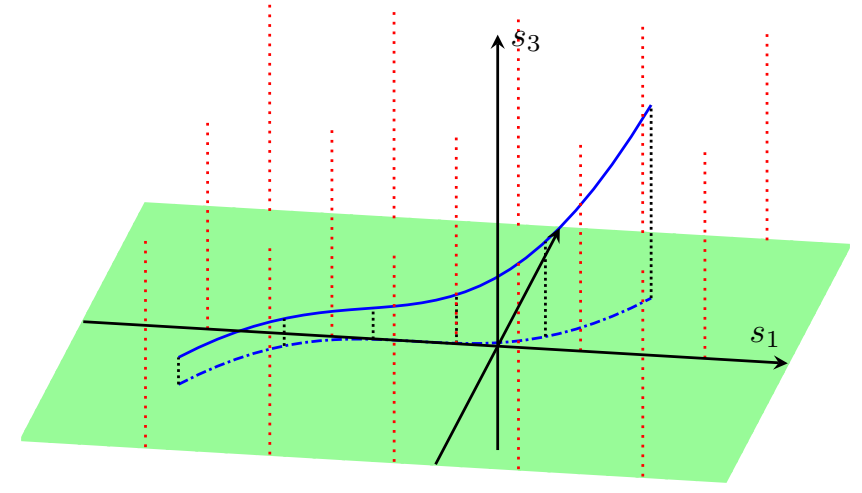

$s_{2}$

transform (8): the terms in (8) are arranged so that this simply means omitting the second line of each of the four pairs of lines.

Then, as plotted schematically in Figure 3, the two Dirichlet boundary conditions (4) at $x=0$ form a one dimensional curve (solid blue line) of allowed values in the three-dimensional centre-stable manifold parametrised by $s_{1}, s_{2}$ and $s_{3}$. Recall $a_{0}$ and $b_{0}$ are the boundary values at $x=0$ from boundary conditions (4). The first two components on the centre-stable manifold $\left(s_{4}=0\right)$ of $(8)$ reveal the microscale constraints on the boundary, upon defining $s_{i}^{0}:=\left.s_{i}\right|_{x=0}$ for $i=1,2,3$,

$$
\left[\begin{array}{l}
a_{0} \\
b_{0}
\end{array}\right] \approx\left[\begin{array}{c}
s_{1}^{0}-s_{2}^{0}+0.25 s_{3}^{0}+1.5 s_{1}^{02}+6 s_{2}^{0^{2}}-1.1 s_{1}^{0} s_{3}^{0}-3.4 s_{2}^{0} s_{3}^{0}-0.035 s_{3}^{0^{2}} \\
s_{1}^{0}+s_{2}^{0}-0.75 s_{3}^{0}-1.5 s_{1}^{0^{2}}-6 s_{2}^{0^{2}}-0.74 s_{2}^{0} s_{3}^{0}+0.25 s_{3}^{0^{2}}
\end{array}\right]
$$

These equations implicitly determine the solid blue curve in Figure 3. To explicitly describe the curve, recall that this is a power series with cubic errors and so we just need to consistently revert the series to give, say, the boundary 
values $s_{1}^{0}$ and $s_{3}^{0}$ as a function of $s_{2}^{0}, a_{0}$ and $b_{0}$. Algebra determines

$$
\begin{aligned}
s_{1}^{0} \approx & \left(0.25 b_{0}-0.29 b_{0}^{2}+0.75 a_{0}-0.63 a_{0} b_{0}+0.18 a_{0}^{2}\right) \\
& +s_{2}^{0}\left(0.5-2.8 b_{0}+3.7 a_{0}\right)+3 s_{2}^{0^{2}} \\
s_{3}^{0} \approx & \left(-b_{0}-0.19 b_{0}^{2}+a_{0}-2.3 a_{0} b_{0}-0.56 a_{0}^{2}\right) \\
& +s_{2}^{0}\left(2-4.6 b_{0}+3.8 a_{0}\right)-5.2 s_{2}^{0^{2}} .
\end{aligned}
$$

Since the slow dynamics in the interior of the domain must lie on the slow manifold $s_{3}=0$, appropriate boundary conditions for the interior dynamics must come from projecting these allowed boundary values onto the slow manifold. Because of the special normal form of the transformed system (9), the slow variables $s_{1}$ and $s_{2}$ evolve independently of the fast variables $s_{3}$ and $s_{4}$, and the appropriate projection is the orthogonal projection along the isochrons $s_{1}$ and $s_{2}$ constant onto the plane $s_{3}=0$ - shown by the red lines in Figure 3. Equation (12a) describes the projected curve in the $s_{1} s_{2}$-plane illustrated by the blue dashed line in Figure 3. Recall from the amplitude definition (7) that $C$ and $s_{1}$ are the same. Hence substituting $s_{1}^{0}=C$ and $s_{2}^{0}=\frac{\partial C}{\partial x}$ into equation (12a) forms the boundary condition at $x=0$ :

$$
\begin{aligned}
& C-\left(0.5-2.8 b_{0}+3.7 a_{0}\right) \frac{\partial C}{\partial x}-3\left(\frac{\partial C}{\partial x}\right)^{2} \\
& \approx\left(0.25 b_{0}-0.29 b_{0}^{2}+0.75 a_{0}-0.63 a_{0} b_{0}+0.18 a_{0}^{2}\right) .
\end{aligned}
$$

This nonlinear Robin boundary condition produces the correct macroscale slowly varying interior domain solutions of the microscale model PDE (1).

\section{A numerical example}

As an example, let the boundary values be $a_{0}=0.2 f(t)$ and $b_{0}=0$ for $f(t)=\tanh ^{2} t$ varying smoothly but quickly from $f(0)=0$ to 1 . Macroscale 
boundary condition (13) gives the macroscale boundary condition at $x=0$ for mean temperature model (2)

$$
C-[0.75 f+0.5] \frac{\partial C}{\partial x}-3\left(\frac{\partial C}{\partial x}\right)^{2}=0.15 f+0.007 f^{2} .
$$

Macroscale boundary conditions on the right One method to derive the macroscale boundary conditions at $x=L$ is to appeal to symmetry. Define a new spatial coordinate $\tilde{x}=\mathrm{L}-\mathrm{x}$ measuring distance from the boundary into the interior, and define new field variables $\tilde{a}(\tilde{x}, t)=-b(x, t)$, $\tilde{b}(\tilde{x}, t)=-a(x, t)$ and therefore $\tilde{C}(\tilde{x}, t)=-C(x, t)$. Then the PDE system (1) is symbolically identical in the tilde and plain variables. But the boundary conditions (4) at the right boundary $x=\mathrm{L}$ are transformed to Dirichlet boundary conditions at $\tilde{x}=0$ of $\tilde{a}(0, t)=-b_{L}$ and $\tilde{b}(0, t)=-a_{L}$. Then the derivation of Sections 2 and 3 apply in the same way to the tilde problem. After computing the macroscale boundary conditions in coordinate $\tilde{x}$ we transform back to the original coordinate $x$.

For example, assume $a_{L}=0$ and $b_{L}=0.2$. The iteration scheme in Section 3 computes the macroscale boundary condition on the boundary $x=L(\tilde{x}=0)$ :

$$
-\tilde{C}-[0.75 f-0.5] \frac{\partial \tilde{C}}{\partial \tilde{x}}+3\left(\frac{\partial \tilde{C}}{\partial \tilde{x}}\right)^{2}=0.15 f-0.007 f^{2} .
$$

By the chain rule $\frac{\partial \tilde{x}}{\partial x}=-1$, and substitute $\tilde{C}(\tilde{x}, t)=-C(x, t)$ into boundary condition (15):

$$
C-[0.75 f-0.5] \frac{\partial C}{\partial x}+3\left(\frac{\partial C}{\partial x}\right)^{2}=0.15 f-0.007 f^{2} .
$$

Numerics verifies the macroscale boundary conditions derivation Figure 2 plots a snapshot of the simulations on microscale model (1) and mean temperature model (2) for two cases: the Dirichlet boundary conditions 
$\mathrm{C}_{0}=\left(\mathrm{a}_{0}+\mathrm{b}_{0}\right) / 2$ and $\mathrm{C}_{\mathrm{L}}\left(\mathrm{a}_{\mathrm{L}}+\mathrm{b}_{\mathrm{L}}\right) / 2$; and our systematic boundary conditions (14) and (16). Using finite differences we convert the system of two PDEs (1) into a system of ODEs. Then Matlab's ode15s applies a variable order method to compute the solution of the system of ODEs (Shampine et al. 1999).

The numerical result is as expected. The macroscale model with systematic boundary conditions (13) model the interior domain microscale dynamics much better than that with heuristic Dirichlet boundary conditions.

\section{Conclusion}

We systematically derived macroscale boundary conditions from microscale Dirichlet boundary conditions. This methodology can be extended to microscale Neumann and Robin boundary conditions. For the microscale Dirichlet boundary conditions, we evaluated the first two components of the centre-stable manifold $(8 \mathrm{a})-(8 \mathrm{~b})$ at $x=0$ to reveal the microscale boundary constraints (11). If the microscale boundary conditions were Neumann, then we would use the last two components, $(8 \mathrm{c})-(8 \mathrm{~d})$. If the microscale boundary conditions were Robin, then we would use linear combinations of the transform (8). The methodology also applies to more general multiscale modelling of PDEs (Roberts 1992).

Future research will generalise the methodology to derive boundary conditions for homogenized models of multiphase materials. Currently, such macroscale boundary conditions are often proposed heuristically without sound mathematical arguments (e.g., Mei and Vernescu 2010, Pavliotis and Stuart 2008). Our systematic approach to macroscale boundary conditions will improve the accuracy the macroscale modelling of multiphase materials. Future applications also include the modelling of spatial patterns. Currently we only know good boundary conditions for a few special cases of the modulation PDEs or of the phase equation. The aim of our systematic approach is to better 
predict boundary effects on the long-time evolution of spatial patterns.

Acknowledgements CC thanks Dr. Tony Miller for his advice and useful discussions, and CSIRO for their support in funding conference and workshop participation.

\section{References}

Bunder, J. E. and Roberts, A. J. (2012), Patch dynamics for macroscale modelling in one dimension. EMAC2011, ANZIAM J. 53:C280-C295. http://journal.austms.org.au/ojs/index.php/ ANZIAMJ/article/view/5074 C17

Chen, C., Roberts, A. J. and Bunder, J. E. (2014), The macroscale boundary conditions for diffusion in a material with microscale varying diffusivities. EMAC2013, ANZIAM J. 55:C218-C234. http://journal.austms.org. au/ojs/index.php/ANZIAMJ/article/view/7853 C17, C19

Dolbow, J., Khaleel, M., Mitchell, J., Pacific Northwest National Laboratory (U.S.) and United States. Dept. of Energy (2004), Multiscale Mathematics Initiative: A Roadmap. Pacific Northwest National Laboratory. http:// multiscale.emsl.pnl.gov/MultiscaleMath3rdworkshop.pdf C17

Kevrekidis, I. G. and Samaey, G. (2009), Equation-free multiscale computation: Algorithms and applications. Annu. Rev. Phys. Chem. 60:321-344. doi:10.1146/annurev.physchem.59.032607.093610 C17

Mei, C. C. and Vernescu, B. (2010), Homogenization methods for multiscale mechanics. World Scientific. http://www.worldscientific.com/ worldscibooks/10.1142/7427 C17, C18, C19, C28

Mseis, G. (2010), The multiscale modeling and homogenization of composite materials. $\mathrm{PhD}$ thesis, The University of California, Berke- 
ley. http://digitalassets.lib.berkeley.edu/etd/ucb/text/Mseis_ berkeley_0028E_10907.pdf C17, C19

Murdock, J. (2003), Normal forms and unfoldings for local dynamical systems. Springer Monographs in Mathematics, Springer. http://link. springer. com/book/10.1007\%2Fb97515 C24

Pavliotis, G. and Stuart, A. (2008), Multiscale Methods: Averaging and Homogenization. Springer. http://www. springer.com/us/book/9780387738284 C17, C18, C28

Ray, N., Muntean, A. and Knabner, P. (2012), Rigorous homogenization of a Stokes-Nernst-Planck-Poisson system. J. Math. Anal. Appl. 390(1):374393. doi:10.1016/j.jmaa.2012.01.052 C19

Roberts, A. J. (1989), Appropriate initial conditions for asymptotic descriptions of the long term evolution of dynamical systems. J. Aust. Math. S. Ser. B 31:48-75. http://journals.cambridge.org/article_ S0334270000006470 C24

Roberts, A. J. (1992), Boundary conditions for approximate differential equations. J. Aust. Math. S. Ser. B 34:54-80. http://journal.austms . org.au/V34/part1/Roberts.html C19, C21, C28

Roberts, A. J. (2013), Macroscale, slowly varying, models emerge from the microscale dynamics in long thin domains. Technical Report. http://arxiv . org/abs/1310.1541 C18

Roberts, A. J. (2014a), Model emergent dynamics in complex systems. Technical report. http://www.maths.adelaide.edu.au/anthony.roberts/ gencm.php C21, C22

Roberts, A. J. (2014b), Model emergent dynamics in complex systems. SIAM. http://bookstore.siam.org/mm20/ C21

Shampine, L. F., Reichelt, M. W. and Kierzenka, J. A. (1999), Solv- 
ing index-1 DAEs in Matlab and Simulink. SIAM Rev. 41(3):538-552. doi:10.1137/S003614459933425X C28

\section{Author addresses}

1. Chen Chen, School of Mathematical Sciences, University of Adelaide, South Australia 5005, Australia.

mailto: chen. chen@adelaide.edu.au

2. A. J. Roberts, School of Mathematical Sciences, University of Adelaide, South Australia 5005, Australia.

mailto: anthony.roberts@adelaide.edu.au

3. J. E. Bunder, School of Mathematical Sciences, University of Adelaide, South Australia 5005, Australia. mailto: Judith.Bunder@adelaide.edu.au 